\title{
Reducción del zinc mediante sulfato de aluminio y superfloc a-110: nivel laboratorio
}

\author{
Reduction zinc by aluminum sulphate and superfloc a-110: \\ laboratory level
}

\author{
Saida Margarita Cuadros Oria ${ }^{1}$, Alex Santiago Uriarte Ortiz ${ }^{2}$, Luz Alexandra Javier Silva ${ }^{3}$ \\ Recibido: 01/07/2020 - Aprobado: 30/10/2020 - Publicado: 30/11/2020
}

\begin{abstract}
RESUMEN
El presente artículo aborda la contaminación por la presencia de zinc en la laguna Pajuscocha, debido a que efluentes mineros que vienen siendo descargados a la laguna con un caudal promedio diario de $52 \mathrm{l} / \mathrm{s}$ aproximadamente, excediendo los límites máximos permisibles para efluentes mineros (> 1,5 mg/l). Para los fines del estudio, se realizaron dos acciones, el primero determinar el pH del caudal y la concentración de zinc del efluente, permitiendo plantear una segunda acción relacionado a la formulación de ocho muestras en función de las variables pH, coagulante Sulfato de Aluminio $\mathrm{Al}_{2}\left(\mathrm{SO}_{4}\right)_{3} \cdot 14 \mathrm{H}_{2} \mathrm{O}$ y floculante Superfloc A-110 a nivel de laboratorio, para la elección del coagulante y el floculante se consideró la eficiencia en costo y beneficio con la finalidad de reducir la concentración del zinc del efluente minero; además de su accesibilidad en el mercado. Finalmente, mediante la ejecución de las dos acciones se logró reducir la concentración del zinc a 0,79 mg/l, mediante las condiciones: coagulante: $6 \mathrm{ml}$, floculante: $1 \mathrm{ml} \mathrm{y} \mathrm{pH}: 8,8$.
\end{abstract}

Palabras clave: Zinc; superfloc A-110; sulfato de aluminio; efluente minero; laguna Pajuscocha.

\begin{abstract}
This article deals with pollution due to the presence of zinc in the Pajuscocha lake, because mining effluents that are being discharged to the lagoon with an average daily flow rate of approximately $52 / \mathrm{s}$, exceeding the maximum permissible limits for mining effluents (>1.5 mg/l). For the purposes of the study, two actions were performed, the first to determine the $\mathrm{pH}$ of the flow rate and the zinc concentration of the effluent, allowing to propose a second action related to the formulation of eight samples based on the variables pH, coagulant Aluminum Sulfate $\mathrm{Al}_{2}\left(\mathrm{SO}_{4}\right)_{3} .14 \mathrm{H}_{2} \mathrm{O}$ and $\mathrm{Superfloc}$ A-110 flocculant at the laboratory level, cost and benefit were considered for the choice of coagulant and flocculant in order to reduce the zinc concentration of the mining effluent; In addition to its accessibility in the market. Finally, by executing the two actions, the concentration of zinc was reduced to $0,79 \mathrm{mg} / \mathrm{l}$, by means of the conditions: coagulant: $6 \mathrm{ml}$, flocculant: $1 \mathrm{ml}$ and $\mathrm{pH}: 8,8$.
\end{abstract}

Keywords: Zinc; superfloc A 110; aluminum sulfate; mining effluent; Pajuscocha Lake.

\footnotetext{
1 Ingeniero Agroindustrial de la Universidad Nacional Federico Villareal. Autor para correspondencia: saidacuadros@gmail.com 2 Ingeniero Ambiental de la Universidad Nacional Federico Villareal. E-mail: alexuriarteortiz@gmail.com 3 Geógrafa de la Universidad Nacional Mayor de San Marcos. E-mail: lualjasigeo@gmail.com
} 


\section{INTRODUCCIÓN}

La Laguna Pajuscocha se encuentra ubicada en el paraje de la Quebrada Tucush y Contonga en el distrito San Marcos, provincia Huari, departamento Ancash a 4038 m.s.n.m, medio ecosistémico donde se alberga cinco especies de flora, dieciocho especies de avifauna y dos especies de peces y fuente natural de agua para el centro poblado de Ccarhuayoc (450 habitantes aproximadamente) ubicado en la parte baja de la Laguna Pajuscocha, dicho recurso es usado con fines agropecuarias.

Los servicios socio-ambientales circundantes que genera la laguna Pajuscocha, recibe el aporte del efluente minero, el cual contiene una elevada concentración de zinc, generando en la flora un desbalance metabólico generalizado en sus células, disminución de frutos y semillas, un crecimiento atrofiado y el amarillamiento de las hojas (clorosis) (Amezcua Romero \& Lara Flores, 2017) y un riesgo de afectación potencial sobre la salud de los pobladores de Carhuayoc, debido a que la ingesta puede producir calambres estomacales, náuseas, vómitos, anemia, daño del páncreas y disminución del tipo de colesterol beneficioso (HDL) en la sangre (ATSDR, 2019).

Por tanto, la presente investigación tiene como objetivo reducir la concentración del zinc del efluente minero mediante la aplicación del coagulante Sulfato de Aluminio $\mathrm{Al}_{2}\left(\mathrm{SO}_{4}\right)_{3} \cdot 14 \mathrm{H}_{2} \mathrm{O}$ y el floculante Superfloc A-110 a nivel de laboratorio, para evitar posibles riesgos de afectación a la flora, fauna y personas mediante el cumplimiento del límite máximo permisible establecido en el Decreto Supremo $\mathrm{N}^{\circ}$ 010-2010-MINAM emitido por el Ministerio del Ambiente. La hipótesis por demostrar es: "El coagulante Sulfato de Aluminio $\mathrm{Al}_{2}\left(\mathrm{SO}_{4}\right)_{3} \cdot 14 \mathrm{H}_{2} \mathrm{O}$ y el Floculante Superfloc A-110 Pajuscocha a nivel de laboratorio alcanza una reducción $(<$ $1,5 \mathrm{mg} / \mathrm{l}$ ) en la concentración del zinc del efluente minero que descarga a la laguna Pajuscocha".

\section{MÉTODO}

El desarrollo metodológico de la investigación comprenden la fase de campo y la fase de laboratorio y en cada una de las fases se ejecutaron diferentes procesos (Ver Figura 1).

El proceso inició con la fase de campo donde se realizó la recolección de la muestra del efluente minero que fue extraída del punto de control denominado: bocamina nivel cero, ubicado en las proximidades de las Quebradas Tucush y Contonga. A partir del punto de control, el efluente minero discurre hacia siete pozas de sedimentación y luego es conducido por un canal sin revestimiento para ser descargado a la Laguna Pajuscocha (Ver Figura 2).

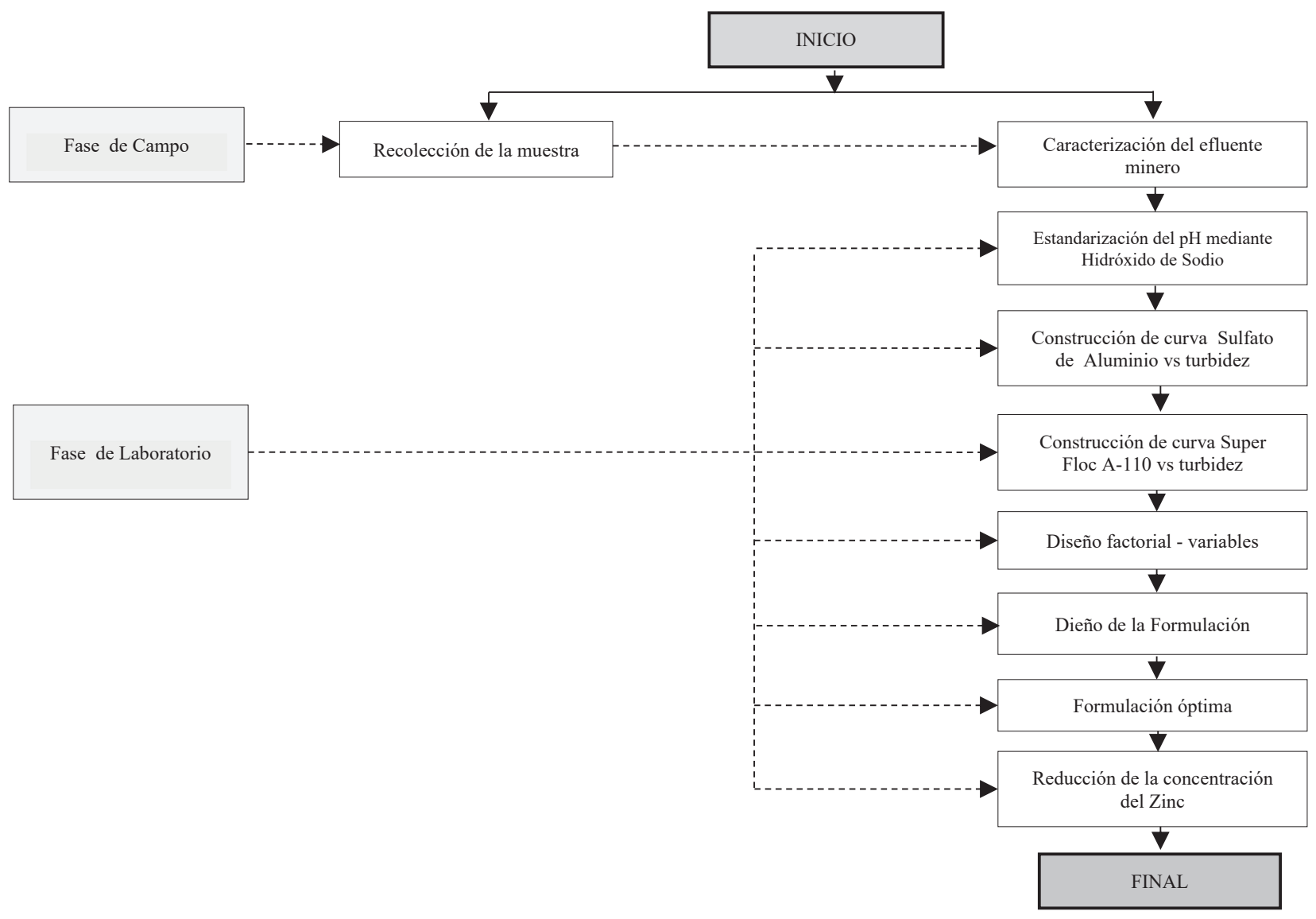

Figura 1. Fases de la Investigación.

Fuente: Elaboración Propia 


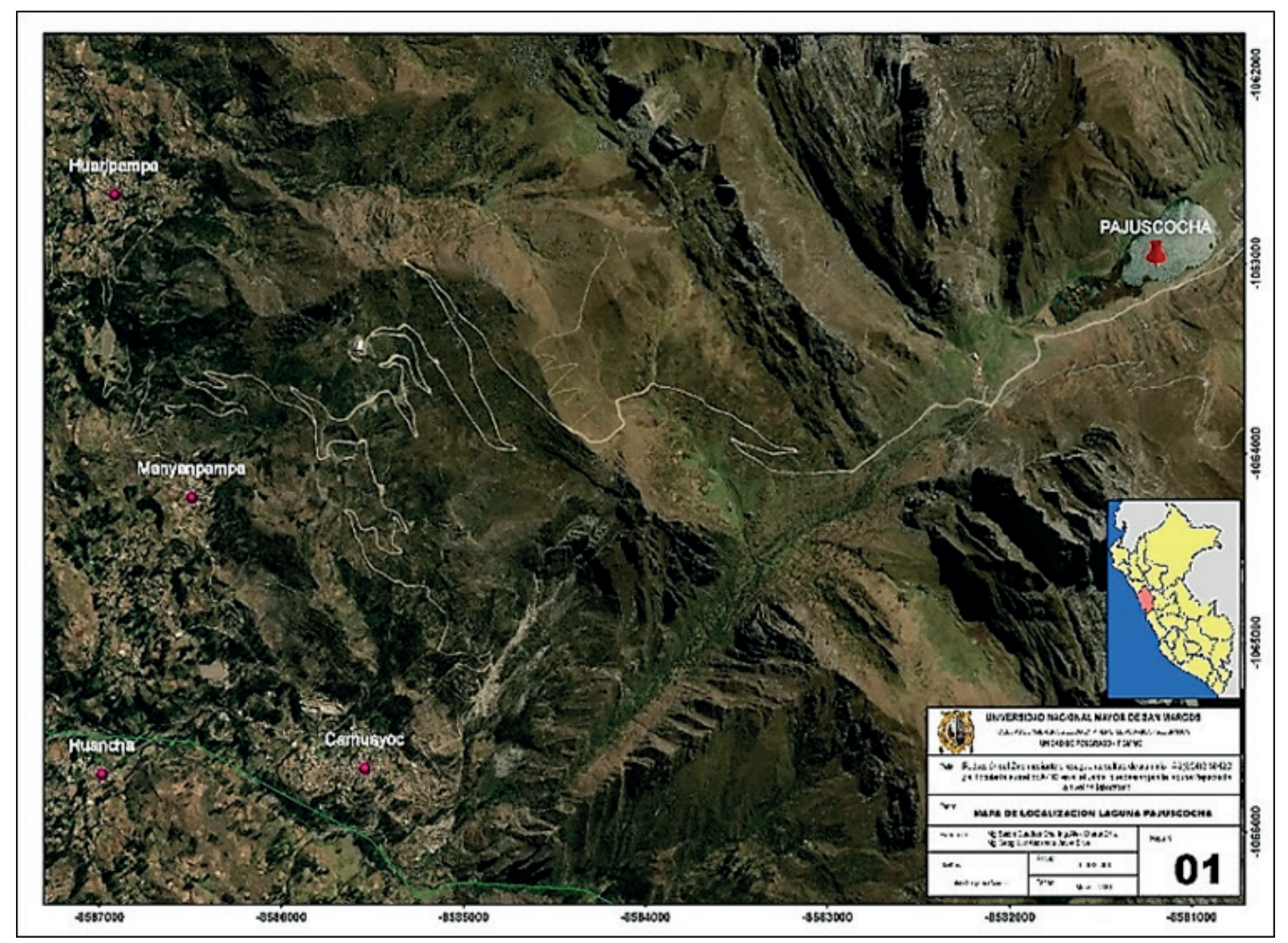

Figura 2. Mapa de ubicación de la Laguna Pajuscocha.

Fuente: Elaboración Propia

En el proceso de recolección de la muestra se midió in situ el $\mathrm{pH}$ utilizando el equipo medidor multiparámetro y la concentración mediante un espectrógrafo por el método de espectrofotometría. La muestra recolectada fue enviada al Laboratorio R-LAB, laboratorio acreditado ante INACAL mediante Registro $\mathrm{N}^{\circ}$ LE-103, considerando los lineamientos establecidos en el Protocolo de Monitoreo de Calidad de Agua de la Autoridad Nacional del Agua-ANA (2016) (Ver Tabla 1).

Tabla 1. Caracterización inicial del efluente minero en la fase de campo

\begin{tabular}{ccc}
\hline \multicolumn{3}{c}{ Características iniciales } \\
\hline pH & Concentración del Zinc & Turbidez \\
\hline 7,80 & $1,79 \mathrm{mg} / \mathrm{l}$ & $60,40 \mathrm{NTU}$ \\
\hline
\end{tabular}

Fuente: Elaboración Propia

Durante la fase de laboratorio se utilizaron como insumos químicos: Hidróxido de Sodio $(\mathrm{NaOH})$ a una concentración de $0,5 \mathrm{~N}$, coagulante Sulfato de Aluminio $\left(\mathrm{Al}_{2}(\mathrm{SO} 4)_{3}\right)$ a $0,2 \%$, floculante Super Floc A-110 a 0,2\% y como materiales: vasos de precipitado, pipeta volumétrica, probeta, tubo de ensayo, rejilla, bureta, matraz, agitador, embudo, balanza, pinzas y cuentagotas.

\section{RESULTADOS Y DISCUSIÓN}

Durante el proceso estandarización del $\mathrm{pH}$ se vertió a la muestra del efluente minero $\mathrm{NaOH} 0,5 \mathrm{~N}$ con el objetivo de acondicionar el valor inicial del $\mathrm{pH}$ dentro del rango básico (8 - 14), debido a que el coagulante reacciona adecuadamente en estado básico, acción que se procedió a realizar en veintidós corridas. De la interacción entre la muestra del efluente minero y el Hidróxido de Sodio, se concluye que el efluente presentó un rango básico de 8,5 (valor inicial) en la Corrida $\mathrm{N}^{\circ} 6$ al añadirse $8,5 \mathrm{ml}$ de $\mathrm{NaOH} 0,5 \mathrm{~N}$ y el valor de 8,8 (valor final) en la Corrida $\mathrm{N}^{\circ} 12$ al verterse $11,5 \mathrm{ml}$ de $\mathrm{NaOH} 0,5 \mathrm{~N}$ (Ver Tabla $2 \mathrm{y}$ Figura 3).

Para la construcción de la curva Sulfato de Aluminio y la variable Turbidez se procedió a realizar doce corridas con los objetivos de (i) disminuir el valor inicial de la turbidez en la muestra del efluente minero añadiendo el Sulfato de Aluminio $\mathrm{Al}_{2}\left(\mathrm{SO}_{4}\right)_{3}$ al $0,2 \%$ y (ii) obtener el volumen adecuado de Sulfato de Aluminio $\mathrm{Al}_{2}\left(\mathrm{SO}_{4}\right)_{3}$ al 0,2 $\%$ para incrementar la turbidez de la muestra del efluente minero. De la relación entre la muestra del efluente minero y el Sulfato de Aluminio $\mathrm{Al}_{2}\left(\mathrm{SO}_{4}\right)_{3}$ al 0,2 \%, se concluyó que el valor inicial de la turbidez disminuyó a 12,4 NTU 
al añadirse $6 \mathrm{ml}$ de $\mathrm{Al}_{2}\left(\mathrm{SO}_{4}\right)_{3}$ al $0,2 \%$. Finalmente, el volumen adecuado para incrementar la turbidez en el efluente minero fue de $9 \mathrm{ml}$ de Sulfato de Aluminio al 0,2\% y manera indirecta la concentración de zinc (Ver Tabla 3 y Figura 4).

La construcción de la curva Super Floc A-110 y la variable Turbidez, se realizó con los objetivos de (i) disminuir el valor inicial de la turbidez en la muestra del efluente minero añadiendo el floculante Super Floc A-110 a $0,02 \%$, y (ii) obtener el volumen adecuado del floculante Super Floc A-110 a 0,02 \% para incrementar la turbidez de la muestra del efluente minero. El resultado de las diez corridas realizadas entre la muestra del efluente minero y el Super Floc A-110, determinó que la turbidez se minimizó al añadirse $6 \mathrm{ml}$ de Super Floc A-110 al 0,02 \% a 18,3 NTU y volumen adecuado del floculante Super Floc A-110 a $0,02 \%$ para aumentar la turbidez fue de $9 \mathrm{ml}$ (Ver Tabla 4 y Figura 5).

En el diseño factorial se consideraron tres variables con ocho combinaciones para determinar la formulación óptima que permita reducir la concentración del zinc en el efluente minero (Ver Tabla 5 y Tabla 6).
Tabla 2. Volúmenes de Hidróxido de Sodio

\begin{tabular}{|c|c|c|}
\hline $\begin{array}{l}\mathrm{N}^{\circ} \text { de } \\
\text { corridas }\end{array}$ & $\begin{array}{l}\text { Volumen (ml) } \\
\mathrm{NaOH} 0.05 \mathrm{~N}\end{array}$ & $\mathrm{pH}$ \\
\hline 1 & 0 & 7.80 \\
\hline 2 & 2.0 & 7.94 \\
\hline 3 & 4.0 & 8.05 \\
\hline 4 & 6.0 & 8.22 \\
\hline 5 & 8.0 & 8.44 \\
\hline 6 & 8.5 & 8.5 \\
\hline 7 & 9.0 & 8.56 \\
\hline 8 & 9.5 & 8.62 \\
\hline 9 & 10.0 & 8.66 \\
\hline 10 & 10.5 & 8.71 \\
\hline 11 & 11.0 & 8.76 \\
\hline 12 & 11.5 & 8.8 \\
\hline 13 & 12.0 & 8.85 \\
\hline 14 & 14.0 & 8.99 \\
\hline 15 & 16.0 & 9.11 \\
\hline 16 & 18.0 & 9.22 \\
\hline 17 & 20.0 & 9.31 \\
\hline 18 & 22.0 & 9.39 \\
\hline 19 & 24.0 & 9.46 \\
\hline 20 & 26.0 & 9.52 \\
\hline 21 & 28.0 & 9.57 \\
\hline 22 & 30.0 & 9.62 \\
\hline
\end{tabular}

Fuente: Elaboración Propia

Tabla 3. Volúmenes de Sulfato de Aluminio

\begin{tabular}{ccc}
\hline $\begin{array}{c}\mathbf{N}^{\circ} \text { de } \\
\text { corridas }\end{array}$ & $\begin{array}{c}\text { Sulfato de } \\
\text { Aluminio a } \\
\mathbf{0 , 2} \%(\mathrm{ml})\end{array}$ & $\begin{array}{c}\text { Turbidez } \\
\text { (NTU) }\end{array}$ \\
\hline 1 & 0 & 60.4 \\
2 & 1 & 38.8 \\
3 & 2 & 28.6 \\
4 & 3 & 23.3 \\
5 & 4 & 21.6 \\
6 & 5 & 17.8 \\
7 & 6 & 12.4 \\
8 & 7 & 22.2 \\
9 & 8 & 22.3 \\
10 & 9 & 22.6 \\
11 & 10 & 31.5 \\
12 & 11 & 35.3 \\
\hline Funte: Elaboración Propia
\end{tabular}

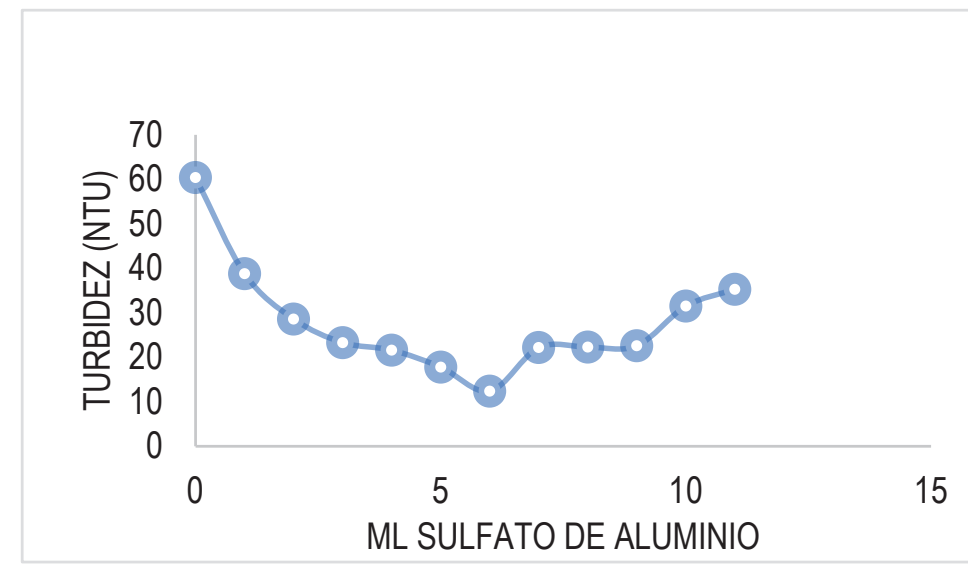

Figura 4. Curva de Coagulación

Fuente: Elaboración Propia 
Tabla 4. Volúmenes de Super Floc A-110

\begin{tabular}{ccc}
\hline $\begin{array}{c}\mathbf{N}^{\circ} \text { de } \\
\text { corridas }\end{array}$ & $\begin{array}{c}\text { Super Floc A-110 } \\
\mathbf{0 , 0 2 \%}(\mathrm{ml})\end{array}$ & $\begin{array}{c}\text { Turbidez } \\
\text { (NTU) }\end{array}$ \\
\hline 1 & 0 & 60.7 \\
2 & 1 & 35.0 \\
3 & 2 & 27.5 \\
4 & 3 & 26.4 \\
5 & 4 & 24.9 \\
6 & 5 & 19.6 \\
7 & 6 & 18.3 \\
8 & 7 & 16.8 \\
9 & 8 & 20.0 \\
10 & 9 & 18.1 \\
\hline
\end{tabular}

Fuente: Elaboración Propia

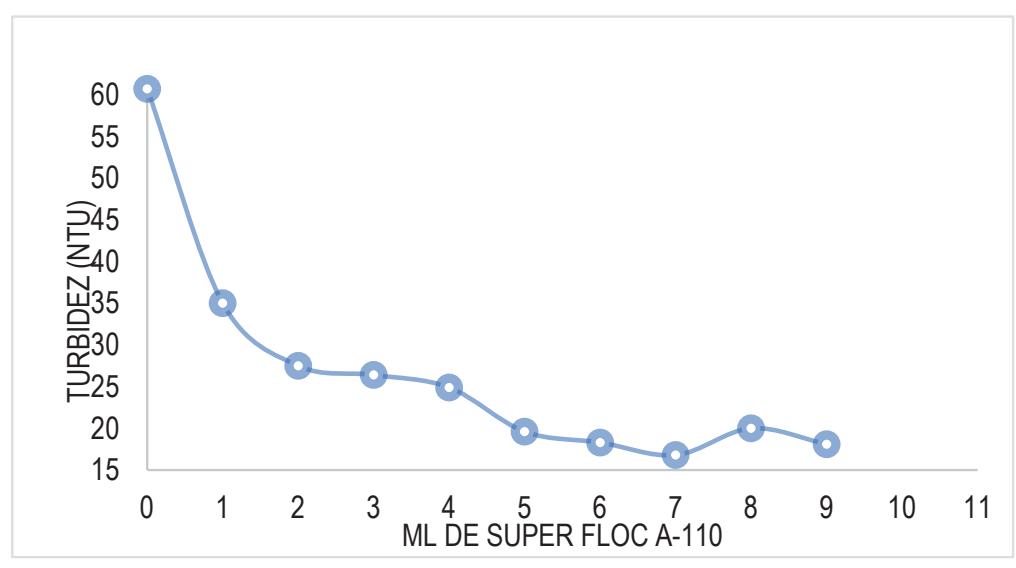

Figura 5. Curva de Floculación

Fuente: Elaboración Propia
Tabla 5. Variables

\begin{tabular}{llll}
\hline & & \multicolumn{2}{c}{ Valores de las variables } \\
\cline { 3 - 4 } \multicolumn{1}{c}{ Ítem } & \multicolumn{1}{c}{ Variables } & \multicolumn{1}{c}{ Valor inicial } & \multicolumn{1}{c}{ Valor final } \\
\hline $\mathrm{A}$ & $\mathrm{pH}$ & 8,5 & 8,8 \\
$\mathrm{~B}$ & Sulfato de Aluminio a $0.2 \% \mathrm{ml}$ & $6 \mathrm{ml}$ & $9 \mathrm{ml}$ \\
$\mathrm{C}$ & Super Floc A-110 a $0.02 \mathrm{ml} \%$ & $1 \mathrm{ml}$ & $5 \mathrm{ml}$ \\
\hline Fuente: & Elaboración Propia & &
\end{tabular}

Tabla 6. Matriz Experimental

\begin{tabular}{cccc}
\hline \multicolumn{4}{c}{ Matriz de experimentos } \\
\hline \multirow{2}{*}{$\mathbf{N}^{\circ}$ de corridas } & \multicolumn{3}{c}{ Variables } \\
\cline { 2 - 4 } & A & B & C \\
\hline 1 & 8,5 & 6 & 1 \\
2 & 8,8 & 6 & 1 \\
3 & 8,5 & 9 & 1 \\
4 & 8,8 & 9 & 1 \\
5 & 8,5 & 6 & 5 \\
6 & 8,8 & 6 & 5 \\
7 & 8,5 & 9 & 5 \\
8 & 8,8 & 9 & 5 \\
\hline
\end{tabular}

Fuente: Elaboración Propia

Finalmente, la formulación óptima se obtuvo a partir de la relación de las variables $\mathrm{pH}$, coagulante y floculante (Ver Tabla 7).

Por tanto, la formulación óptima se compuso de: Sulfato de Aluminio $\mathrm{Al}_{2}\left(\mathrm{SO}_{4}\right)_{3}$ al 0,2\%: $6 \mathrm{ml}$, Super Floc A-110 a 0,02 \%: $1 \mathrm{ml}$ e Hidróxido de Sodio $(\mathrm{NaOH})$ a $0,5 \mathrm{~N}: 11,5 \mathrm{ml}$, permitiendo reducir la concentración del zinc del efluente minero a $0,79 \mathrm{mg} / \mathrm{l}$, resultado que se encuentra dentro de lo exigido por la norma referencial correspondiente a efluentes minero.

Vilca Cabana (2018) utilizó el coagulante Sulfato de Aluminio $\mathrm{Al}_{2}\left(\mathrm{SO}_{4}\right)_{3}$ al $10 \%(200 \mathrm{mg} / \mathrm{l})$ y floculante Reactivo MPP50 (200 mg/l), formulación que redujo la concentración inicial de zinc de $110 \mathrm{mg} / \mathrm{l}$ a $1,80 \mathrm{mg} / \mathrm{l}$ de los efluentes mineros de la unidad minera El Cofre; sin embargo, dicha formulación no permitió no exceder el límite maximo permisible $(<1,5 \mathrm{mg} / \mathrm{l})$ establecido en el Decreto Supremo $\mathrm{N}^{\circ} 010$ emitido por el Ministerio del Ambiente (2010), a pesar de que dicha formulación utilizó el coagulante Sulfato de Aluminio; en este sentido el coagulante Sulfato de Aluminio es más compatible floculante Super Floc A-110 y no con el floculante Reactivo MPP50; toda vez que en la presente investigación dicha combinación permitió reducir la concentración de zinc antes de su descarga a la Laguna Pajuscocha a 0,79 ,g/l y no exceder los límite maximo permisible establecidos en la normativa mencionada.

Tabla 7. Formulación óptima para la reducción de la concentración del zinc

\begin{tabular}{ccccc}
\hline $\mathrm{N}^{\circ}$ & $\begin{array}{c}\mathrm{A} \\
\mathbf{p H}\end{array}$ & $\begin{array}{c}\mathrm{B}: \\
\text { Sulfato de } \\
\text { Aluminio (ml) }\end{array}$ & $\begin{array}{c}\text { C } \\
\text { Super Floc } \\
\text { A-110 (ml) }\end{array}$ & $\begin{array}{c}\text { Concentración de } \\
\text { Zinc (mg/l) }\end{array}$ \\
\hline 1 & 8.5 & 6 & 1 & 1,7915 \\
2 & 8.8 & 6 & 1 & 0,7910 \\
3 & 8.5 & 9 & 1 & 1,8044 \\
4 & 8.8 & 9 & 1 & 0,8887 \\
5 & 8.5 & 6 & 5 & 1,7761 \\
6 & 8.8 & 6 & 5 & 1,2571 \\
7 & 8.5 & 9 & 5 & 2,1181 \\
8 & 8.8 & 9 & 5 & 2,4119 \\
\hline
\end{tabular}

Fuente: Elaboración Propia

En relación con la metodología planteada por Ceras Cuadros \& Ochoa León (2013) donde se utilizó Hidróxido de Sodio $(\mathrm{NaOH})$ a $0,1 \mathrm{~N}$ para neutralizar el contenido de zinc del efluente minero antes de ser vertidas al río Rímac. El Hidróxido de Sodio $(\mathrm{NaOH})$ a $0,1 \mathrm{~N}$ neutralizó el valor inicial $(6,59)$ del $\mathrm{pH}$ ácido a los valores de 7,03 y 8,52 añadiendo $8 \mathrm{ml}$ a $13 \mathrm{ml}$, respectivamente; difiere a nuestro planteamiento metodológico que utilizó Hidróxido de Sodio $(\mathrm{NaOH})$ a $0,5 \mathrm{~N} \mathrm{ml}$ que neutralizó a un valor inicial 7,80 de $\mathrm{pH}$ básico a 8,5 y 8,8 virtiendo $8,5 \mathrm{ml}$ y $11,5 \mathrm{ml}$ respectivamente. 
De acuerdo a la investigación planteada por (Apaza Asqui, 2015) se advierte que utilizó el Hidróxido de Sodio $(\mathrm{NaOH})$ a $0,1 \mathrm{~N}$ para neutralizar el contenido de zinc $(2,38 \mathrm{mg} / \mathrm{l})$ de las aguas del río T'oroQ'ocha de la ciudad de Juliaca (Puno). El insumo mencionado neutralizó el valor inicial $(7,8)$ del $\mathrm{pH}$ a los valores de 7,65 y 10,75 añadiendo $0,5 \mathrm{ml}$ a $5 \mathrm{ml}$ de Hidróxido de Sodio $(\mathrm{NaOH})$ a $0,1 \mathrm{~N}$, respectivamente; discrepa de nuestro planteamiento metodológico que utilizó Hidróxido de Sodio $(\mathrm{NaOH})$ a $0,5 \mathrm{~N}$ ml que neutralizó a un valor inicial 7,80 de $\mathrm{pH}$ a 8,5 y $8,8 \mathrm{pH}$ virtiendo $8,5 \mathrm{ml}$ y $11,5 \mathrm{ml}$ respectivamente, siendo el presente estudio demandante de mayor cantidad de Hidróxido de Sodio $(\mathrm{NaOH})$ a comparación el estudio planteado por (Apaza Asqui, 2015).

Ceras Cuadros \& Ochoa León (2013) utilizó el coagulante Tricloruro de Hierro $\left(\mathrm{FeCl}_{3}\right)$ al $0,1 \%$ para minimizar la turbidez del efluente minero. El efluente minero presentó una turbidez inicial de 372,20 NTU, el cual se redujo al verterse de $8 \mathrm{ml}$ hasta $14 \mathrm{ml}$ del Tricloruro de Hierro $\left(\mathrm{FeCl}_{3}\right)$, a un valor final de turbidez de 3,04 NTU; sin embargo, la presente investigación al verter $6 \mathrm{ml}$ del coagulante Sulfato de Aluminio $\mathrm{Al}_{2}$ (SO4)3 al 0,2\% logro reducir el valor inicial de la turbidez de 60,4 NTU a 12,4 NTU; comparación que permite detallar que el Sulfato de Aluminio es mas eficiente que el Tricloruro de Hierro para reducir la turbidez en el efluente minero. Se determinó utilizar 6 ml de Super Floc A-110 a 0,02 \% para disminuyir la turbidez de la muestra del efluente minero a 18,3 NTU y aumentar el contenido de Super Floc A-110 a 0,02 \% a 9 $\mathrm{ml}$ para aumentar la turbidez obteniendo un valor de 18,1 NTU, en comparación; (Ceras Cuadros \& Ochoa León, 2013) utilizó el floculante Polychem $0,05 \%$, logrando reducir la turbidez vertiendo mayor volumen de floculante desde $7 \mathrm{ml}$ hasta $10 \mathrm{ml}$, obteniendo valores 0,07 NTU y 0,05 NTU respectivamente.

La concentración del zinc mediante la formulación de Ceras et. al (2013): floculante Polychem 0,05\%: $7 \mathrm{ml}$, coagulante Tricloruro de Hierro $\left(\mathrm{FeCl}_{3}\right)$ a $0,1 \%$ : $14 \mathrm{ml}$ e Hidróxido de Sodio $(\mathrm{NaOH})$ a $0,1 \mathrm{~N}: 8 \mathrm{ml}$, logrando minimizar la concentración del zinc del efluente minero a $0,05 \mathrm{mg} / \mathrm{l}$; y cuyo resultado se diferencia debido a que se utilizan mayores volúmenes de coagulante, floculante y neutralizante para reducir la concentración de zinc, acción que implica mayor costo de inversión (Ver Figuras 6 - 9).

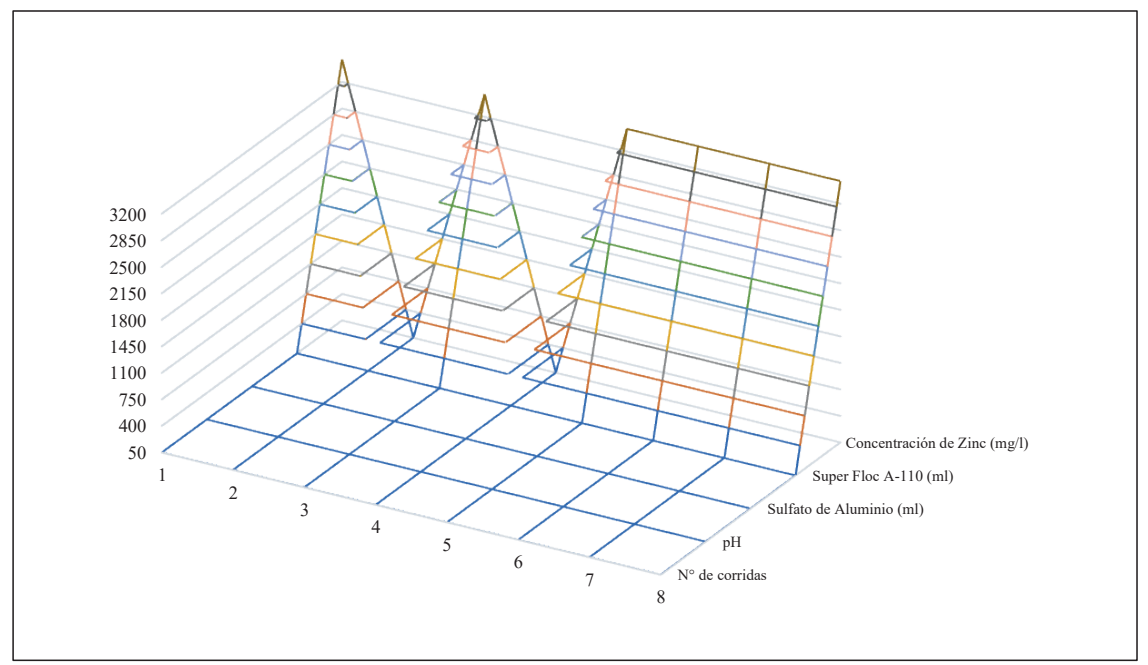

Figura 6. Gráfico de Respuesta en relación a la formulación óptima para la reducción de la concentración del zinc

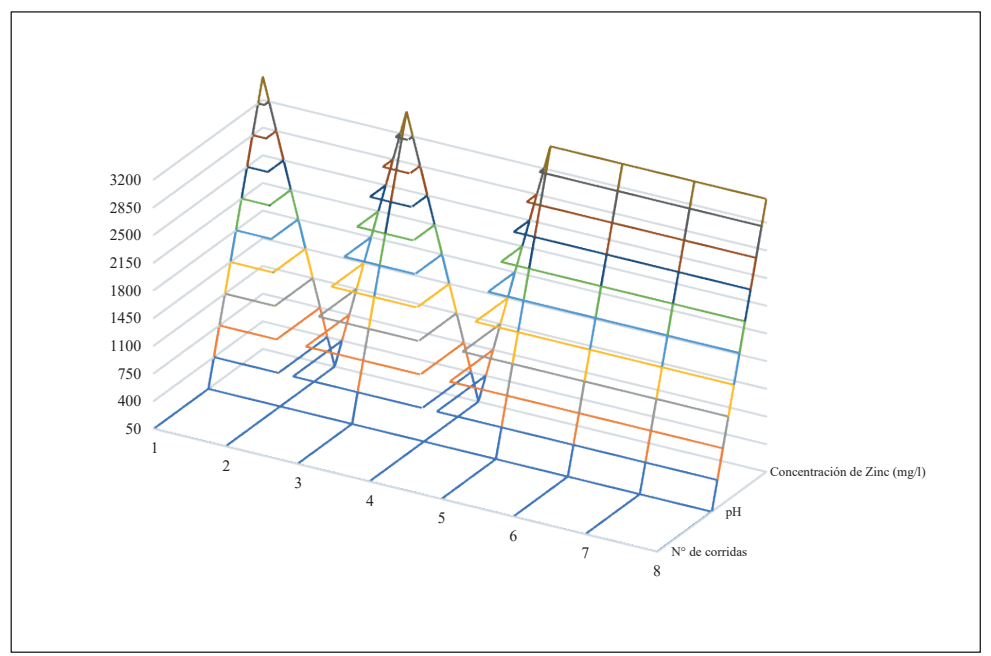

Figura 7. Gráfico de Respuesta en relación de la variable potencial de hidrógeno y la concentración de zinc 


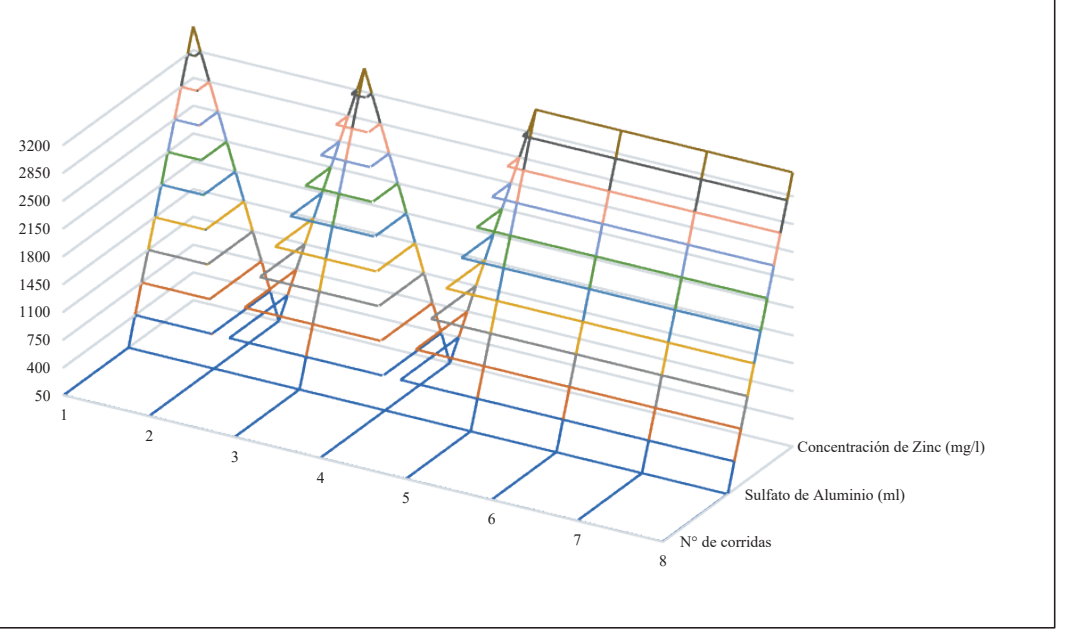

Figura 8. Gráfico de Respuesta en relación de la variable sulfato de aluminio y la concentración de zinc

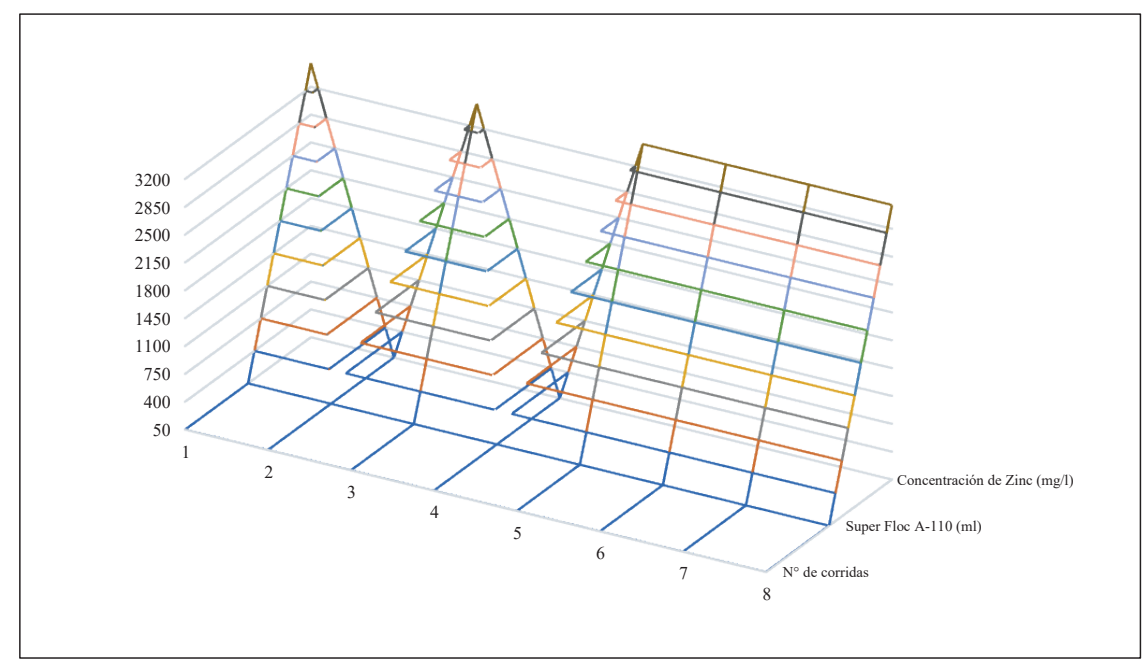

Figura 9. Gráfico de Respuesta en relación de la variable Super Floc A-110 y la concentración de zinc

\section{CONCLUSIONES}

La hipótesis se comprobó mediante la formulación del Sulfato de Aluminio $\mathrm{Al}_{2}\left(\mathrm{SO}_{4}\right)_{3}$ al 0,2 \% y Super Floc A-110 a $0,02 \%$, logrando reducir la concentración del zinc en el efluente minero que descarga a la Laguna Pajuscocha a un valor de $0,79 \mathrm{mg} / \mathrm{l}$, resultado que no excede los límites máximos permisibles $(1,5 \mathrm{mg} / \mathrm{l})$ establecido en el Decreto Supremo $\mathrm{N}^{\circ} 010$ del MINAM-Ministerio del Ambiente (2010).

La relación entre el coagulante Sulfato de Aluminio $\mathrm{Al}_{2}\left(\mathrm{SO}_{4}\right)_{3}$ al $0,2 \%(6 \mathrm{ml})$ y $\mathrm{pH}$ básico $(8,8)$ permitieron reducir la concentración del zinc presente el en efluente minero, relación que contribuye a no exceder el valor establecido en la normativa referencial peruana.

El valor del floculante Super Floc A-110 a 0,02 \% y $\mathrm{pH}$, que contribuyeron en la remoción del zinc del efluente minero es la correspondiente a $1 \mathrm{ml} \mathrm{y} 8,8$ respectivamente.

\section{AGRADECIMIENTOS}

Nuestro agradecimiento a las autoridades y docentes de la Facultad de Ingeniería Geológica, Minera, Metalúrgica y Geográfica de la Universidad Nacional Mayor de San Marcos, por su apoyo en la presente investigación.

\section{REFERENCIAS}

Amezcua Romero, J. C., \& Lara Flores, M. (2017). El Zinc en las Plantas. Ciencia. https://www.atsdr.cdc.gov/es/phs/ es_phs60.html

Apaza Asqui, D. W. (2015). Remoción de metales Plomo (Pb) y Zinc (Zn) de las aguas del rio T'oro Q'ocha por precipitación alcalina en la Ciudad de Juliaca, Puno. Universidad Nacional Del Altiplano. http://repositorio.unap.edu.pe/ handle/UNAP/10984\%0Ahttp://repositorio.unap.edu.pe/ handle/UNAP/13304 
ATSDR - Agencia para sustancias tóxicas y el registro de enfermedades. (2019). Resúmenes de Salud Pública - Cinc (Zinc). https://www.globalfinancingfacility.org/sites/gff_ new/files/Tanzania_One_Plan_II.pdf

Autoridad Nacional del Agua-ANA. (2016). Protocolo Nacional para el Monitoreo de la Calidad de los Recursos Hídricos Superficiales aprobado mediante R.J. $N^{\circ}$ 010-2016-ANA. https://www.ana.gob.pe/normatividad/rj-no-010-2016-ana-0

Ceras Cuadros, C., \& Ochoa León, H. G. (2013). Remoción de Zinc de las aguas de mina en el nivel 3900 de la compañia minera Los Quenuales - Unidad de producción Yauliyacu a nivel de laboratorio. http://repositorio.uncp.edu.pe/ bitstream/handle/UNCP/3712/Ceras Cuadros-Ochoa León. pdf? sequence $=1 \&$ is Allowed $=y$
MINAM-Ministerio del Ambiente. (2010). DS $\mathrm{N}^{\circ}$ 010-2010-MINAM: Aprueban límites máximos permisibles para la descarga de efluentes líquidos de actividades minero - metalúrgicas. In El Peruano (pp. 424114-424117). http:// www.minam.gob.pe/wp-content/uploads/2013/09/ds 0102010-minam.pdf

Vilca Cabana, S. R. (2018). Recuperación de Cadmio y Zinc en la Unidad Minera El Cofre con el reactivo MPP50 por coagulación-floculación. Universidad Nacional Del Altiplano. http://repositorio.unap.edu.pe/handle/ UNAP/4061 